\title{
Sul calcolo dell'ampiezza originaria di un'onda presente in una serie temporale causale e stazionaria di dati equi- spaziati attraverso Io studio di uno spettro di potenza
}

(A technique of power spectra analysis to evaluate original amplitude of harmonic components in stationary random time series of equispaced data)

\author{
P. RANDI - M. E. RoNCHI SELva (")
}

Riceruto il 26 Gennaio 1973

Riassunto. - ln un precedente lavoro ( ${ }^{4}$ ) gli Autori hanno descritio un programma che permette il calcolo dello spettro di potenza di una scric temporale casuale e stazionaria di lati equispaziati. Nel presente lavoro viene esposta una tecniea che permette di risalire al valore sell'ampiezza originaria di un'onda presente nella serie di slati e viene oflerta la possibilità al rieereatore tli eseguire un'analisi automatica delle curatteristiche delle onde più significative estruendone le eorrispondenti ampiezze, sotto determinate condizioni da lui stesso poste.

11 modo di utilizzo e la specifica delle eondizioni sono descritte nel paragrafo "Ltilizzazione del programma - INPUT-OLTPUT", esposto più sotto.

SUmmary. - A prevision work (4) describeda program which allows the computation of power spectrum for stationary random time-series of equispaced data. In the present work we describe a methorl to evaluate the original wave amplitude of harmonie terms in these series. This tecnique allows authomatical analysis of the most sirnificant features of principal harmonic components in the serie, under some user conditions. In the section "Utilizzazionc id programma - INPUT-OLTPLT" we describe these conditions and how the program SPECTRA is accessible and utilizable.

(*) Centro di Cakolo Interuniversitario dell'Italia Nord-Oriontale. Casalecchio ti Reno (Bulogna) - Italia. 


\section{If, P'ROGRAMHA SPECTRA}

Il programma SPJECTRA permette al ricerentore ti fare l'analisi spettrale su una serie temporale stazionaria di rati casuali eq equispaziati di qualunque lunghezza slopo aver fatio precelentemente sulla stessi queste operazioni:

a) Si esegue un esame preliminare allo scopo tli ripristinare la sequenza tenporale corretta qualora, o per la presenza di dati ritemuti spuri, comungue originati, o per la mancanza di dati, la serie in INPIT non risulta equispaziata.

b) Si riporta detta seric nelle contizioni di media zero.

Alla serie $T(t)$ quale risulta dalle operazioni sopra descritte viene applicata la finestra:

$$
D_{2}(t)=\frac{\mathrm{L}}{2} \cdot\left(1+\cos \frac{\pi t}{T}\right) \quad \operatorname{con}: t !<T
$$

allo scopo ali eliminare le deformazioni sulle frequenze dovute alla. limitatezan della serie tlei dati.

Viene quindi calcolata la funzione oli antocorrelazione:

$$
F(\tau)=\frac{i}{N^{\top}-\tau} \sum_{i=1}^{N} b_{2}(t) X(t) D_{2}(t+\tau) X(t+\tau)
$$

col ribardo $\tau$ che va da zero al massimo fissato alad ricercatore. Infine viene calcolato lo spetiro di potenza tramite lat:

$$
\varphi(\omega)=2 \sum_{\tau=0}^{\tau \operatorname{lnax}} H^{T}(\tau) \cos \omega \tau
$$

con tutte le curatteristiche e disponibilità esposte nel lavoro già citato.

I problemi su eni gli Autori hamo fissato la loro attenzione sono i soguenti: data l'altezza del piceo dello spettro, corrisponslente a una certa frequenza, quale l'ampiezza effettiva dell'onda originaria che lat generato quella banda spettrale; qual'è inoltre la dipendenza Aellorilinata spettrale, ossia dellaltezan dei picchi dello spettro di potenza, dal ritario massimo $\tau_{\text {max }}$ seelto dal ricercatore e infine come i posibile eseguire automaticamente nua analisi di $\varphi(0)$ per ricavarne informazioni sulle ampiezze, dopo aver stabilito nu opportuno criterio di significativitì per i massimi relativi ahe si presentano nello spettro. 


\section{J RISTLTATI DELL'LDAGIXE}

Merlinte l'uso del programma SPECTRA gli Antori hanno indagato sulle variazioni di altezza dei pichi dello spettro di potenza in funzione delle caratteristicle della serie data, e cioè al variare del numero tei dati e dellampiezza reale tlelle ourle originarie, la cui sovrapposizione costituisce la serie in esame.

Fissato che la funzione di autocorrelazione è stata calcolata lino al massimo ritardo compatibile con la lunghezza della serie, i risultati dell'indagine sono mostrati in fig. 1.

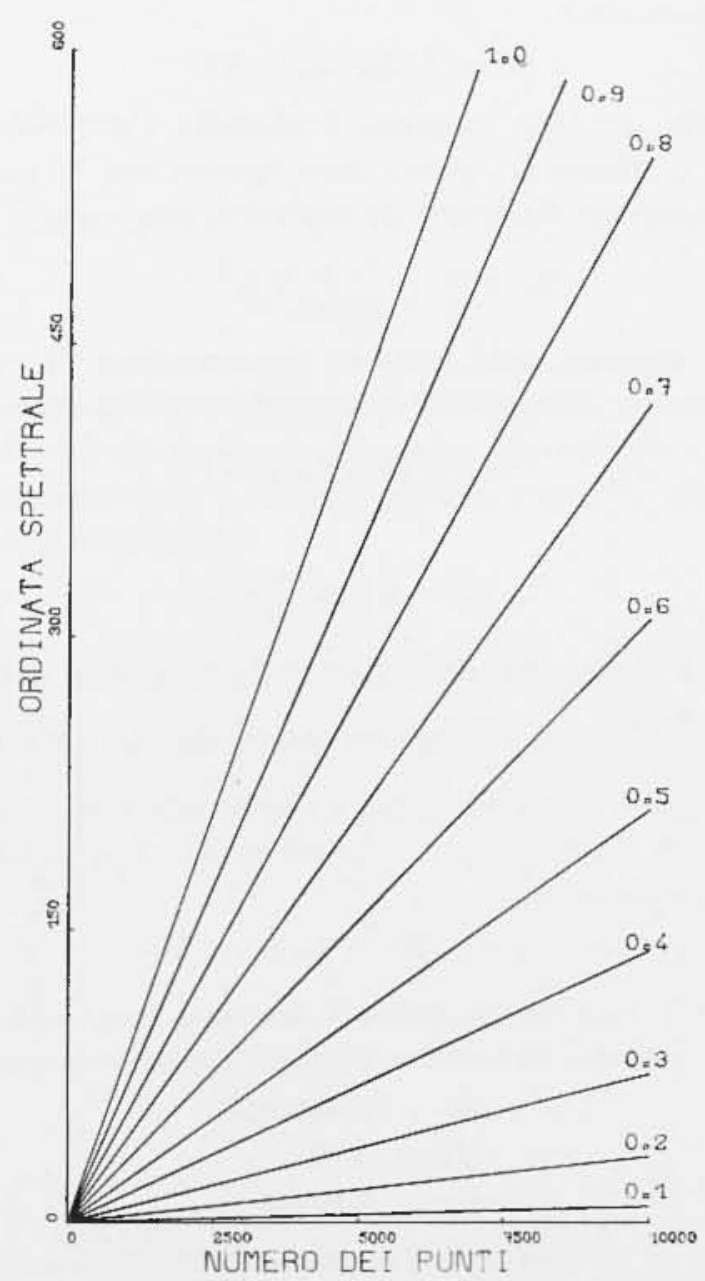

Fig. 1 
Come si può velere, esiste una dipendenza lineare fra l'altezza dei picehi $\varphi\left(\omega_{i}\right)$ corrispondenti a una certa frequenza e la lunglezza della serie, ad ampiezza originaria costante, del tipo:

$$
\varphi\left(\omega_{1}\right)=a_{1} N
$$

essendo $N$ il numero degli individui componenti la serie ed $\alpha_{1}$ un opportumo coefficiente.

Tutite queste rette, passanti jer l'origine, hanno il coefficiente angolare $a_{1}$ che è funzione dell'ampiezza dell'onda originaria, della estensione temporale della serie data nonchè del ritardo massimo $\left(\tau_{\max }\right)$, scelto dal ricercatore a seconda del problema tratitato; per cui si puó affermare clıe:

$$
a_{1}-f\left(A_{1}, \tau_{\max }, N\right) .
$$

Ora poiclı̀ ciò che interessa è ricavare l'ampiezza, una volta determinate le altezze dei picchi dello spettro per le varie frequenze presenti, conoscendo il numero di individui componenti la serie data e noti come

$$
\alpha_{1}=\varphi\left(\omega_{1}\right) / N
$$

i coefficienti angolari delle rette di ajpartenenza di ciascuna delle ordinate spettrali, si è cerento di esplicitare la funzione

$$
a_{1}=f\left(A_{1}, \tau_{\max }, N\right)
$$

eome

$$
A_{\mathrm{i}}=f_{1}\left(\alpha_{\mathrm{i}}, \frac{\tau_{\max }}{N}\right) .
$$

Quando $\tau_{\max }$ è il massimo consentito dalla lunghezza della serie data, cioè quando $\frac{\tau_{\max }}{\bar{N}} \sim 1$, la migliore interpolazione dei valori ottenuti per $f_{1}$ ì una parabola passante per l'origine con asse parallelo all'asse delle ascisse, il cui ramo per $y$ positive (che ì quanto interessa) d̀ deserit to dall'equazione

$$
A_{1}=\sqrt{K_{1}+K \cdot a_{1}}-K_{3}
$$

dove $a_{1}$ sono $\mathrm{i}$ suldetti coefficienti angolari, ricavabili come soprat esposto, e le costanti numericle valgono rispettivamente

$$
\begin{aligned}
& \boldsymbol{K}_{1}=0.0002020 \\
& \Pi_{2}=12.06 \\
& K_{3}=0.01421
\end{aligned}
$$

In figura 2 si mostra l'andamento della funzione sopra scritta quandlo, come detto, $\tau_{\max }$ è il massimo consentito dalla serie dei clati. 
Una ulteriore indagine s stata fatta per individuare il legame tra le ordinate spettrali e i ritardi massimi, $\tau_{\max }$, lissati per il calcolo.

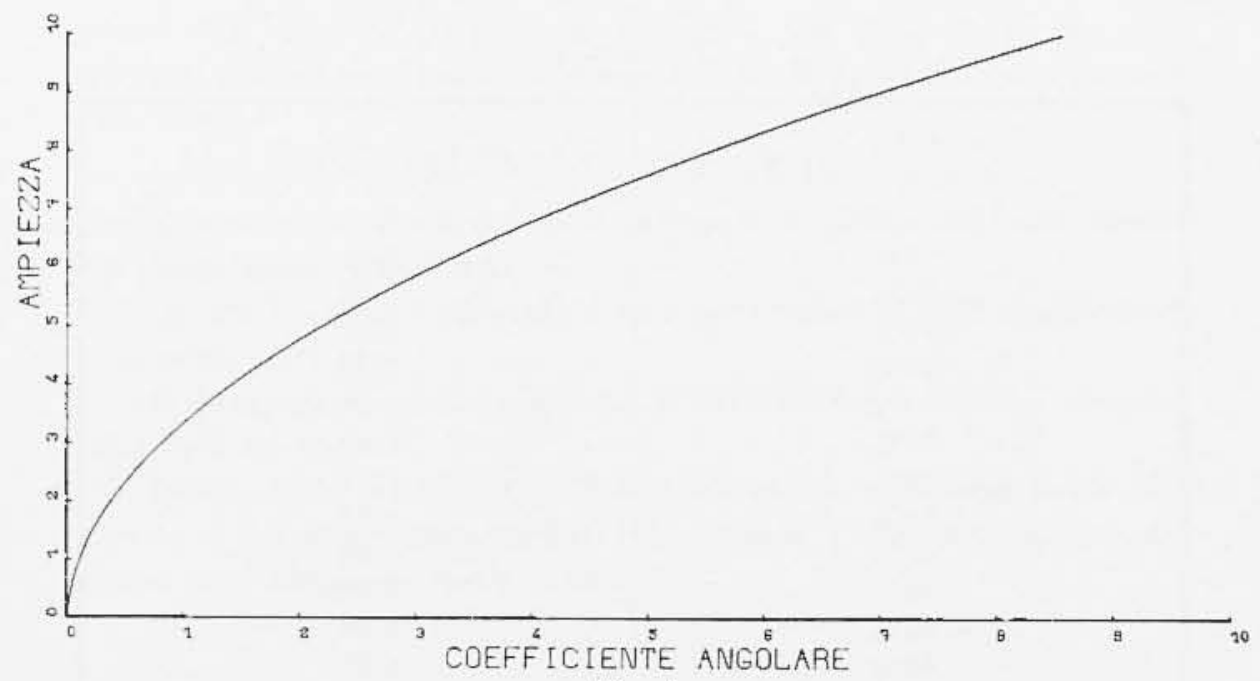

Fin. 2

Si \& trovato che, indipendentemente dallampiezza dell'onda che ha generato quella banda spettrale, il variare del rapporto $\tau_{\text {max }} / \mathcal{H}$ genera una variazione concorde $1 / \gamma$ dell'ordinata spettrale con un legame del tipo mostrato in fig. 3.

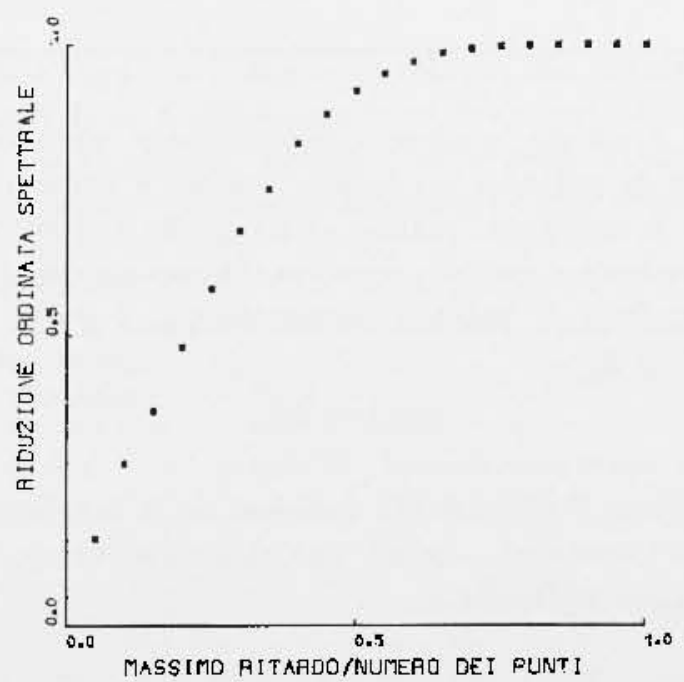

Fig 3 
In Tabella 1 sono esplesse percentalmente queste variazioni in lunzione di $\tau_{\max } / N$.

TABHELA ]

\begin{tabular}{|c|l|}
\hline$\tau / N$ & $1 / \gamma$ \\
\hline $5 \%$ & \\
$10 \%$ & 0.15 \\
$15 \%$ & 0.28 \\
$20 \%$ & 0.37 \\
$25 \%$ & 0.48 \\
$30 \%$ & 0.58 \\
$35 \%$ & 0.68 \\
$40 \%$ & 0.75 \\
$45 \%$ & 0.83 \\
$50 \%$ & 0.88 \\
$55 \%$ & 0.92 \\
$60 \%$ & 0.95 \\
$65 \%$ & 0.97 \\
$70 \%$ & 0.985 \\
$75 \%$ & 0.993 \\
$80 \%$ & 0.997 \\
$85 \%$ & 0.999 \\
$90 \%$ & 0.9998 \\
$95 \%$ & 1.0000 \\
$100 \%$ & 1.0000 \\
& 1.0000 \\
\hline & \\
\hline
\end{tabular}

Infine si è voluta rendere completamente automatica l'analisi di uno spettro di potenza. Tal tecnica seguita is stata quella che tradizionalunente is applicata quando si lia a clie verlere con una serie di dati indipendenti e casuali: sono considerati signifieativi tutti quei picehi dello spettro di potenzi corrispondenti a $p\left(\omega_{1}\right)$ il cui valore lisulta maggiore di

$$
\varphi\left(\omega_{1}\right)+2 \sigma
$$

In conchusione il ricercatore, qualsiasi sia la lnnghezza tlella scric, qualsiasi sia il ritarlo $\tau_{\text {mux }}$ usato, può risalire al valore ilell"umpiezza tell'onda originale mediante la:

$$
A\left(\omega_{1}\right)=\sqrt{K_{1}+K_{2} \frac{\gamma \varphi\left(\omega_{1}\right)}{N}}-K_{3}
$$


relazione rhe è stata programmata e inserita come implementazione nel programma SPECTRA.

Qualora si preferisea sostiluire al eriterio di significativita dei pieclia dello spettro di potenza utilizzato dal programma un altro ritenuto più consono (ad es. il criterio di Walker) ì sufficiente serivere una routine

\section{FUNCTION TEST (PTH)}

forzandone la compilazione ed il caricamento prima diel caricamento del programma SPECTRA.

Questa funzione d diversa da zero per i valori di PII significativi, zero negli altri casi.

Il programma chiama guesta FUSCTION per tutii i massimi relativi dello spettro.

Quella della FUNCTION TEST ì l'unica variazione lecita alla struttuma del programmu SPECTRA, affinchè i risultati relativi alla stima delle ampiezze siano validi.

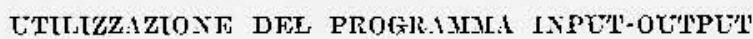

Viene qui descritta la sequenza di schele parametri necessarie allesecuzione flel programmat. Il contenuto di questo paragrafo in parte ripete ciò che è stato detto nel lavoro già vitato in preserenza (4) in parte dipenfle dalla introduzione tli nuve implementazioni nel programma SPICCTRA el ì quinti una novita rispetto al precedente seritto.

Le schere contenenti i purametri relativi alla eventuale ristuntturazione dei dati letti e allelaborazione della serie in ingresso sono due e devono essere sempre presenti. Ia presenza di altre selrede può essere necessaria sotto particolari contizioni come scritto sotto.

1a seheda FORMAT (212, I1, 212, I1, 2F5.0,15, 3F10.0,I1, 18X, 6I1)

\begin{tabular}{|c|c|c|c|}
\hline Colonna & $\begin{array}{c}\text { Yome della } \\
\text { variabile }\end{array}$ & Tipo & Descrizione \\
\hline 1.2 & MT & intero & 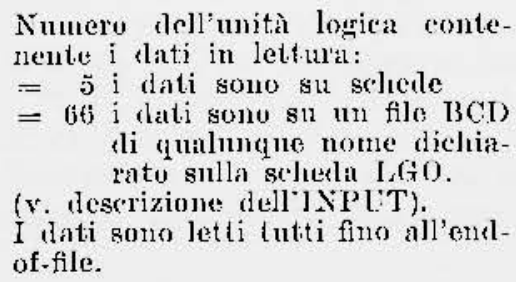 \\
\hline
\end{tabular}


(segue 1a seheda)

\begin{tabular}{|c|c|c|c|}
\hline Colonna & $\begin{array}{l}\text { Nome della } \\
\text { variabile }\end{array}$ & Tipo & Descrizione \\
\hline $3-4$ & $\mathrm{NN}$ & intero & $\begin{array}{l}\text { Numero dei dati per scheda o } \\
\text { record (nassimo } 80 \text { ). }\end{array}$ \\
\hline 5 & I7E R I & intero & $\begin{aligned} \text { se }=0 & \text { i dati nulli sono signifieativi } \\
\text { se } \neq 0 \text { i dati nulii rappresentano } & \\
& \text { dati mancanti o errati } \\
& \text { verranno sostituiti nel cor- } \\
& \text { so del programma. }\end{aligned}$ \\
\hline $6-7$ & GAPI $\left(^{*}\right)$ & intero & $\begin{aligned} & \text { se }=0 \text { nessun gap in lettura nei } \\
& \text { dati } \\
& \text { se } \neq 0 \text { intliea il numoro di volte } \\
& \text { che si lebbono saltare i } \\
& \text { dati in lettura, dati clic } \\
& \text { retranno sostituti sueces- } \\
& \text { sivanente onde ripristina- } \\
& \text { re la sequenza temporale } \\
& \text { della scrie (massimo } 99 \text { ). }\end{aligned}$ \\
\hline $8-9$ & GAPE (*) & intero & 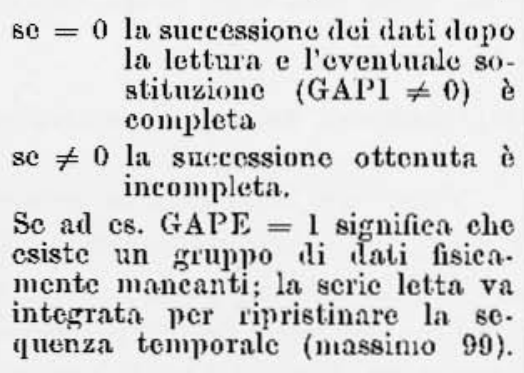 \\
\hline 10 & crrrus & intero & $\begin{aligned} & \text { se }=0 \text { nei gaps viene inserita la } \\
& \text { media della serie lotta } \\
& \text { se } \neq 0 \text { nei gaps vengono inseriti } \\
& \text { sati elaborati mediante in- } \\
& \text { terpolazione lineare usu- } \\
& \text { fruendo del metolo di } \\
& \text { elhiusura. }\end{aligned}$ \\
\hline 11.15 & PERC & reale & $\begin{array}{l}\text { Differenza percentuale richiesta } \\
\text { dal metolo di chiusura. }\end{array}$ \\
\hline
\end{tabular}

(*) $\Lambda \mathrm{i}$ parametri GAPI, GAPE ì associato un numero $n=$ GAPI + GAPE di sehede che devono seguire la scheda parametri suecessiva alla scheda in esane. Cinseuna sli queste schede contiene due valori interi con FORMAT (2110) rappresentanti il numero d'oldine, nella sequenza tempo. rale ta claborare, dei dati validi immediatamente esterni ad ogni gap da integrare. Relativamente a GAPI $=1$ comparira una scheda contenente al es. 25 (col. 9-10) e 42 (col. 19-20); questo significa che in lettura i dati dal $26^{\circ}$ al $41^{\circ}$ sono non signifieativi e anslramo opportunamente sostiluiti. Queste sehele devono essere orelinate per valori ereseenti dei limiti dei gaps. 
(segue 1a scheda)

\begin{tabular}{|c|c|c|c|}
\hline Colonna & $\begin{array}{l}\text { Nome della } \\
\text { variabile }\end{array}$ & Tipo & Deserizione \\
\hline $16 \cdot 20$ & $\mathrm{~T}$ & reale & $\begin{array}{l}\text { Semilunghe\%za della finestra D2 } \\
\text { (in unitâ di ascissa). }\end{array}$ \\
\hline $21 \cdot 25$ & TAUMAX & intero & $\begin{array}{l}\text { Massimo ritardo nel caleolo della } \\
\text { funzione di autocorrelazione (mas- } \\
\text { simo 9999). }\end{array}$ \\
\hline $26-35$ & NIMIX & reale & $\begin{array}{l}\text { Valore minimo delle frequenze con. } \\
\text { sideraie nel calcolo dlello spettro } \\
\text { di potenza. }\end{array}$ \\
\hline $36-45$ & NIMAX & reale & Valore massimo delle frequenze. \\
\hline $46 \cdot 55$ & TONT & reale & $\begin{array}{l}\text { Passo di frequenza, ossia diffe- } \\
\text { renza in ascissa fra } 2 \text { punti con- } \\
\text { secutivi dello spettro di potenza. }\end{array}$ \\
\hline 56 & $\mathrm{DN} 2$ & intero & $\begin{array}{l}\text { Intica quante volle si vuole appli- } \\
\text { carc ai dati la finestra D2. }\end{array}$ \\
\hline 75 & IFPRINT & intero & $\begin{array}{l}=1 \text { viene stamputa la tabella } \\
\text { contenente, per ogni riga, la } \\
\text { coppia di ascissa e ordinata } \\
\text { spettrali di tutti } \mathrm{i} \text { punti dello } \\
\text { spettro di potenza. } \\
=0 \text { la precerlente tabella non } \\
\text { viene stampata. }\end{array}$ \\
\hline 76 & IFFUNC & intero & $\begin{array}{l}\text { = } 1 \text { viene stampata la tabella } \\
\text { contenente, per ogni riga, } \\
\text { ascissa e ordinata spettrali } \\
\text { noncile ampicz\% originaria } \\
\text { dei punti massimi relativi } \\
\text { signilicativi dello spettro. } \\
=0 \text { la precetente tabella non } \\
\text { viene stampata né calcolata. }\end{array}$ \\
\hline 77 & IFX & intero & $\begin{array}{l}=0 \text { non vengono eseguiti nè il } \\
\text { orafico né la perforazione } \\
\text { tella seric data. } \\
=1 \text { viene eseguito solo il grafico; } \\
=2 \text { vienc eseguita solo la per- } \\
\text { forazione; } \\
=3 \text { vengono esegniti sia il grafico } \\
\text { sia la perforazione. }\end{array}$ \\
\hline 78 & $1 \mathrm{~F} \mathrm{~F}$ & intero & $\begin{array}{l}\text { Analogo a } I F X \text { per la scric dopo } \\
\text { la D2. }\end{array}$ \\
\hline 79 & IFTAU & intero & $\begin{array}{l}\text { Analogo a } 1 F \mathrm{X} \text { per la funzione di } \\
\text { autocorrelazione. }\end{array}$ \\
\hline 80 & IFPHI & intero & $\begin{array}{l}\text { Analogo a } 1 \mathrm{FX} \text { per lo spettro dij } \\
\text { potenza. }\end{array}$ \\
\hline
\end{tabular}


2: scheda - FORAIT $(8 \mathrm{A10})$

\begin{tabular}{|c|c|c|c|}
\hline Colomma & $\begin{array}{c}\text { Nomedella } \\
\text { variabile }\end{array}$ & Tipo & Descrizione \\
\hline $1-80$ & IFORM & $\begin{array}{c}\text { alfa } \\
\text { numerico }\end{array}$ & $\begin{array}{c}\text { Contiene il formato di lettura nel } \\
\text { normale linguagrio Fortran, con- } \\
\text { prese le parentesi esterme. }\end{array}$ \\
\hline
\end{tabular}

Il programma SPECTRA iे disponibile già compilato su un Permanent File ed i richiamabile e ntilizzabile metiante la seguente sequenza di scherle:

a) nel caso in eni i dati sono contenuti sn nastro:

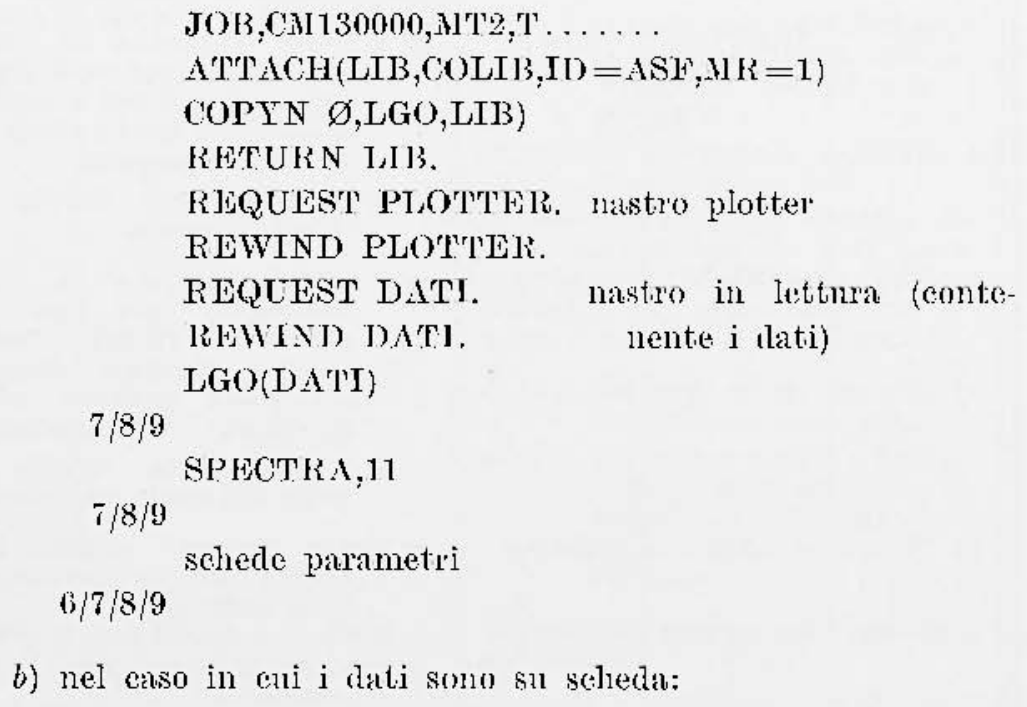

b) nel caso in cai i dati sono su soleda:

JOB,CAI130000, MIT1,T . . .

$\left.\left.\triangle \mathrm{T}^{\prime} \mathrm{ACH}(\mathrm{T} \mathrm{I} B, \mathrm{COI}] \mathrm{B}, \mathrm{IT}\right)=\Lambda \mathrm{SF}, \mathrm{MR}=1\right)$

COPYN $(\varnothing, \mathrm{LG}(0, \mathrm{LIB})$

RHTURN LIB.

REQUEST PLOT'TER, nastro plotter RHWINI) PI,OTTER.

LGO. 


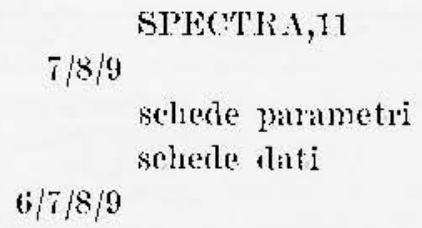

is opportuno forse a questo punto aggiungere unt osservazione sui nomi di tile riservati dal programma SPECTRA e sulla sucesessione dei parametri della scherla LGO manijolabili rventualmante per l'esecuzione dlel programma.

SPECTRA utilizad internamente i files TAPE1, TAPE:, TAPE3, TAPEA, TAPlis, TAPJA, TAPET, TAPEt6; i quali non flevono essere altrimenti usati in un jols che rsegma SPECTRA.

I pammetri delinibili allatto dellesecuzione mediante la scherla LGO sono:

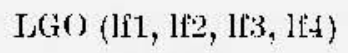

If́1 = nome alel file contenente i dati in lettuar (quanto questi non sono su scliesta) (dlelault name $=$ DATT)

lf: $=$ nome del file contenente l'uscita binaria del plotter folefanlt. name = PIoTTTliR)

If 3 = defiult name =- IXTPT

$\mathrm{li} \Psi=$ default name $=$ OUTPUT.

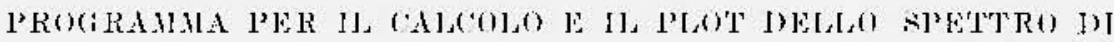
TOTENZA

Costanti nate per l'elahorazime

Numero dei dati letti $=8000$

Numero dei jumti dolla $132=5999$

Massime ritardo, $=5995$

Freq. milt. $=0$.

Freq. max. $=3.00000000 \mathrm{~F}-01$

P'asso = 1.00000)

Valori massinos o minimo dolle strie

Fal. massino $=4.654094 \mathrm{~J} 6 \mathrm{\textrm {E }}+00$ Val. minimo $=-3.70119278 \mathrm{E}: 00$

Val. massino $=4.06428324 \mathrm{E}-1-00$ Vil, minimo $=-4.11630707 \mathrm{E}+00$

Val. missimu $=7.48305788 \mathrm{~F}-0 \mathrm{I}$ Val, minimo $=-4.59271637 \mathrm{~F}-0 \mathrm{I}$

Val, massinto $=2.42406+82 \mathrm{E}+02$ Val, minimo $=-1.14494097 \mathrm{E}+01$ 


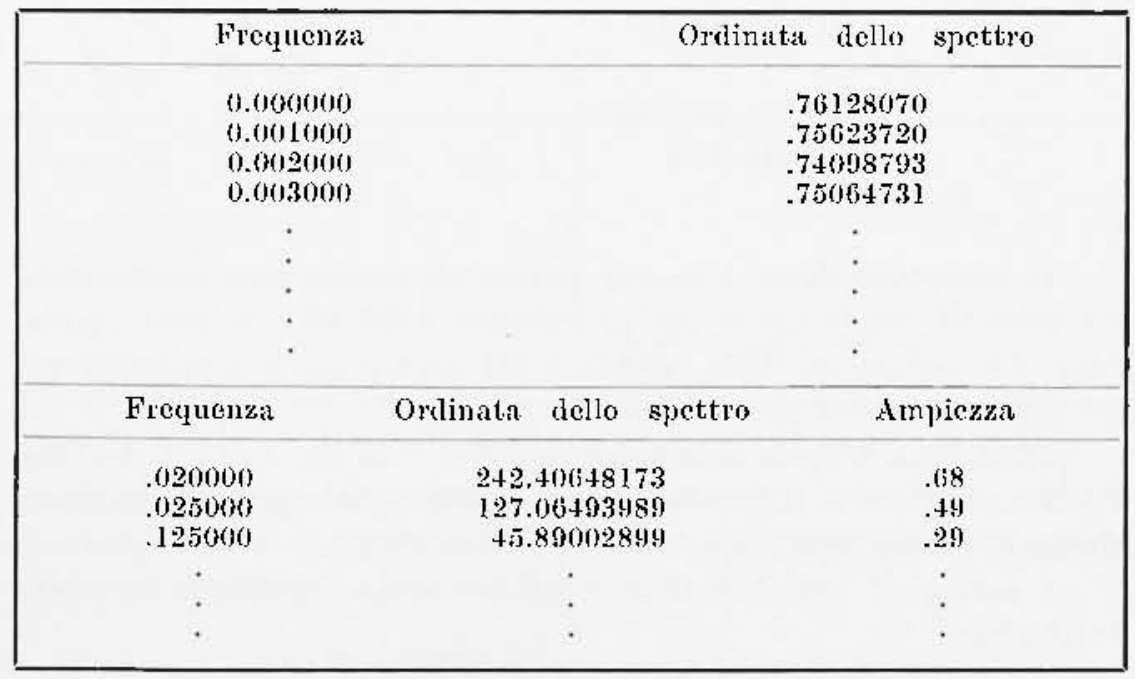

Fisr. $4 b$

E possibile ottenere, su scelta dell'Utente, altre 2 tabelle, l'una contenente i valori di ascissa ordinata spettinale di tutti i punti dello spettro, l'altra contenente ascissa e ordinata spettrale e ampiezza originaria relative ai punti di massimo relativo, significativi secondo i criteri esposti nei paragrafi precedenti (vedi es. in fig. $4 \mathrm{a}, \mathrm{b}$ ).

Per qunto concerne l'uscita su tabulato, essa contiene sempre una tabella con i seguenti dati:

a) numero e valori massimo e minimo della serie dei dati da elaborare; la $\mathrm{D} 2$;

b) numero e valore minimo e massimo dei dati della serie dopo

e) valore del massimo ritardo e valori massimo e minimo della funzione di antocorrelazione;

d) frequenza minima, massima e passo di frequenza usati per il calcolo dello spettro di potenza, valore massimo e minimo dell'ordinata spettrale.

\section{BIBJIOGRAFIA}

(1) Benpat J. S. and Pifrsol A. G., 1966. - Measurement and Analysis of Random Datn. "John Wiley \& Son, Inc. n, New York. 


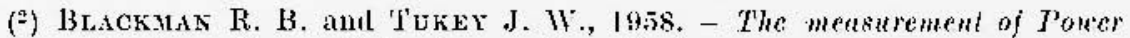
Speetra. "Dover Publ. Ine, ", New York.

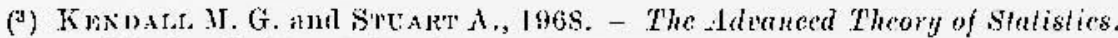
"Charles Gribin \& Co. Jald. ", Jumbun.

(1) Raxin P., Roxcin Skiva M. E., 1972. - Sullo spettro di protenza. " J'Elaborazione automalica n, 1, 2.

(5) Solodovnikov V. V., 1965. - Dinnmique Statistifue des Systemes Lineaires de Commande automatigue. " $\mathrm{D}$ nnod n, Paris.

(i) YAgLom A. A., 1962. - An Introduction to the Theory of Stationary Random Functions. "P'rentice-Ilall, Ine, Englewood Clifis", New Jerscy. 